\title{
МИРОВОЙ ОПЫТ ОЦЕНКИ ЭФФЕКТОВ ОТ РЕАЛИЗАЦИИ ПРОЕКТОВ В СФЕРЕ ГОРОДСКОГО ОБЩЕСТВЕННОГО ТРАНСПОРТА
}

\author{
(c) 2021 Батукаев Артем Темирович \\ аспирант департамента мировой экономики и международного бизнеса \\ Финансовый университет при Правительстве Российской Федерации, Россия, Москва \\ E-mail: batukaev.at@gmail.com
}

В статье раскрыты основные зарубежные методики проведения оценки влияния проектов в сфере общественного транспорта на городскую экономику. Проведено исследование, в рамках которого были выявлены основные эффекты от реализации проектов в сфере общественного транспорта. Даны основные понятия и определения при оценке проектов в сфере городского транспорта.

Ключевые слова: общественный транспорт, экономический эффект, социальный эффект, анализ «выгода-затраты», экологический эффект, агломерация, пассажирские перевозки, урбанизация.

В мире в условиях постоянной урбанизации, а следовательно, и увеличения количества агломераций, происходит постоянный прирост пассажирских транспортных потоков. Проекты в области транспорта влияют на обширный спектр отраслей экономики: за счет формирования заказа промышленности (построение новых магистралей, разработка отдельных полос движения для общественного транспорта, производство подвижного состава) и сферы услуг (ремонт дорожного покрытия, услуги перевозки, услуги по лизингу и страхованию подвижного состава). В связи с этим возникает необходимость проведения оценки социально-экономических эффектов от реализации проектов в сфере городского общественного транспорта, так как на основе оценки социально-экономических эффектов решаются вопросы развития сферы общественного транспорта, оптимизации транспортных схем, цифровизации и модернизации общественного транспорта.

Весь мировой транспортный сектор переживает перемены глобального масштаба, вызванные рядом факторов. К ним относятся технологические факторы, изменения в структурах управления транспортом, усугубление экологических проблем, а также необходимость обеспечения мобильности и доступности горожан независимо от социального статуса или уровня дохода. Для реализации социальноэкономически оправданных инвестиций в транспортную систему, особенно там, где задействованы новые технологии, необходим новый взгляд на экономику и инвестиции, который формируется на основе применения результатов комплексной оценки социально-экономических факторов [3].

Пассажирский городской транспорт является ключевой частью городской инфраструктуры и объединяет в себе массовый общественный транспорт (перевозящий пассажиров по определенным маршрутам, т.е. уличный, например: трамвай, троллейбус, автобус, и внеуличный скоростной, например: метрополитен, скоростной трамвай, наземные электрички), легковой автомобильный транспорт (например: такси, ведомственные и личные автомобили), транспорт последней мили (электросамокаты, сигвеи и пр.) [1].

В представленной диаграмме (рис. 1) отображена структура пассажирских перевозок на территории РФ за 2019 г. Согласно диаграмме, большую часть в перевозках занимает личный транспорт $(63,4 \%)$, в то время как доля общественного агломерационного транспорта (автобусы, трамваи, троллейбусы, пригородные поезда, метрополитен) занимает лишь треть от общего объема предоставляемых услуг (33,9\%). Можно сделать вывод о том, что городские поездки (на личном и общественном транспорте) составляют большую часть всех перевозок (более 80\%), создает предпосылки

В мировой практике широко распространены три подхода для оценки и выбора реализуемых проектов в транспортной среде: анализ «выгоды-затраты», анализ совокупного экономического эффекта, многофакторный анализ.

Использование анализа «выгоды-затраты» (cost-benefit analysis, CBA) структурами, ответственными за сферу общественного транспорта и инфраструктуры, происходит редко. Шведскими учеными в работе «Использование анализа «выгоды-затраты» органами общественного 


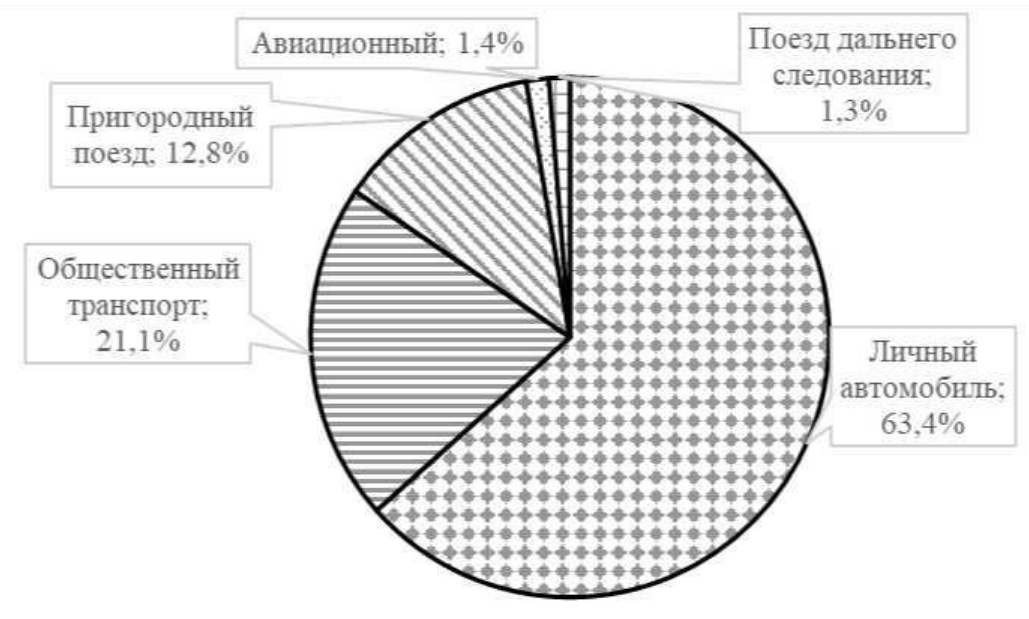

Рисунок 1. Доля видов транспорта в перевозках пассажиров РФ в 2019 г. Источник: составлено автором по данным [2].

транспорта на практике» было выяснено, что большинство организаций не используют метод анализа СВА. Те организации, которые прибегают к данной методике анализа, используют ее в основном для оценки эффективности получения инвестиций в платежные системы, или же для крупных изменений на линиях и маршрутах. Самой большой причиной для отказа от данного метода анализа являются недостаток компетенций сотрудников управляющей организации в сфере проведения анализа СВА и большая зависимость от других видов анализов принятия решений [4].

Применение методики СВА обусловлено целями оценки эффектов. Если проверка необходима государственному органу, то соответствующему органу, необходима оценка, проведенная в соответствии с утвержденными методиками и на основе легкопроверяемых, так называемых, «прозрачных» данных. В данном случае, как видно по мировому опыту, используется методика СВА.

Анализ совокупного экономического эффекта представляет собой оценку влияния реализованных и разрабатываемых проектов с точки зрения нескольких показателей: прирост выпуска предприятий, чистый приход доходов, прирост инвестиций, создание новых рабочих мест. Данный метод анализа включает в себя также несколько видов эффектов. Первая категория представляет собой прямые эффекты - то есть экономию на операционных расходах. К ним можно отнести экономию рабочего времени, экономию расходов на эксплуатацию транспортных средств. Второй категорией эффектов (косвенными эффектами) можно считать те, которые не оказывают прямого влияния на денежные потоки, а направлены на социальную сферу.
В условиях конкуренции за ограниченные ресурсы правительствам необходимо продемонстрировать соотношение цены и качества новых инфраструктур. СВА - это традиционный подход к обеспечению благосостояния, позволяющий продемонстрировать ценность использования государственных средств. СВА учитывает материальные затраты и выгоды для пользователей, выгоды и затраты от внешних эффектов. Однако этот подход к оценке благосостояния не позволяет определить распределение воздействия деятельности общественного транспорта с точки зрения пространственного расположения, времени или конкретных секторов экономики, а также не отражает все воздействия на экономику региона. Анализ совокупного экономического эффекта показывает такие изменения с точки зрения рабочих мест, компенсаций операционных расходов и объема производства. Изменения экономического воздействия в значительной степени являются языком, используемым политиками для объяснения преимуществ инвестиций и лучшего понимания их сути электоратом. Эти подходы дополняют друг друга, отвечают на разные вопросы и могут быть использованы вместе для более целостной оценки стоимости изменений в инфраструктуре общественного транспорта [5].

Многофакторный анализ (multi-criteria analysis, MCA) - специфичный метод, используется для сравнения проектов и дальнейшего составления их рейтингов. Таким образом, полная мультикритериальная оценка проектов происходит благодаря количественным рейтингам, которые включают в себя проведенное сравнение эффектов по различных направлениям, и качественным оценкам. Данный метод позволя- 
ет оценивать наиболее широкий спектр эффектов, как позитивных, так и негативных. Многофакторный анализ позволяет анализировать и оценивать такие категории, как: конкурентоспособность бизнеса, эффективность логистических систем, доступность рабочих мест и рынки экспорта.

Последовательное принятие решений требует структурированной и систематической оценки преимуществ и недостатков различных возможностей выбора. Для транспортных проектов, а также для оценки вариантов транспортировки были разработаны и эффективно применяются различные многокритериальные методы в дополнение к традиционному анализу эффективности затрат и выгод. На основе широкого обзора литературы сделан вывод, что методы МСА применяются в основном для оценки вариантов транспортировки, а не транспортной политики или проектов, а наиболее часто используемым методом МСА в решении проблем транспортного сектора является аналитический иерархический процесс [6].

Если говорить о моделях, используемых для оценки будущих эффектов, они могут разительно отличаться и включать в себя множество ал- горитмов вычислений и наборы входных данных. Наиболее используемыми моделями для оценки влияния развития инфраструктурных проектов являются: модели межотраслевого баланса (МОБ), городские транспортные модели (LUTI - land-use transport interaction models), модели общего равновесия (CGE - computable general equilibrium models), эконометрические модели.

Повышение спроса на общественный транспорт отражает экономическую активность фирм, агломераций и частных лиц. Но изменение транспортной инфраструктуры оказывает воздействие на экономическое развитие. Данное воздействие можно разделить на: прямое воздействие - связанное с транспортной системой, и на косвенное - связанное с влиянием транспортной отрасли на связанные сектора экономики. Также существуют и другие воздействия, например, экологическое. На рис. 2 приведена концепция возникновения социальноэкономических эффектов. Из представленной схемы можно сделать вывод о том, что данные эффекты порождаются инфраструктурными эффектами, которые в свою очередь определяются параметрами среды и параметрами проекта.

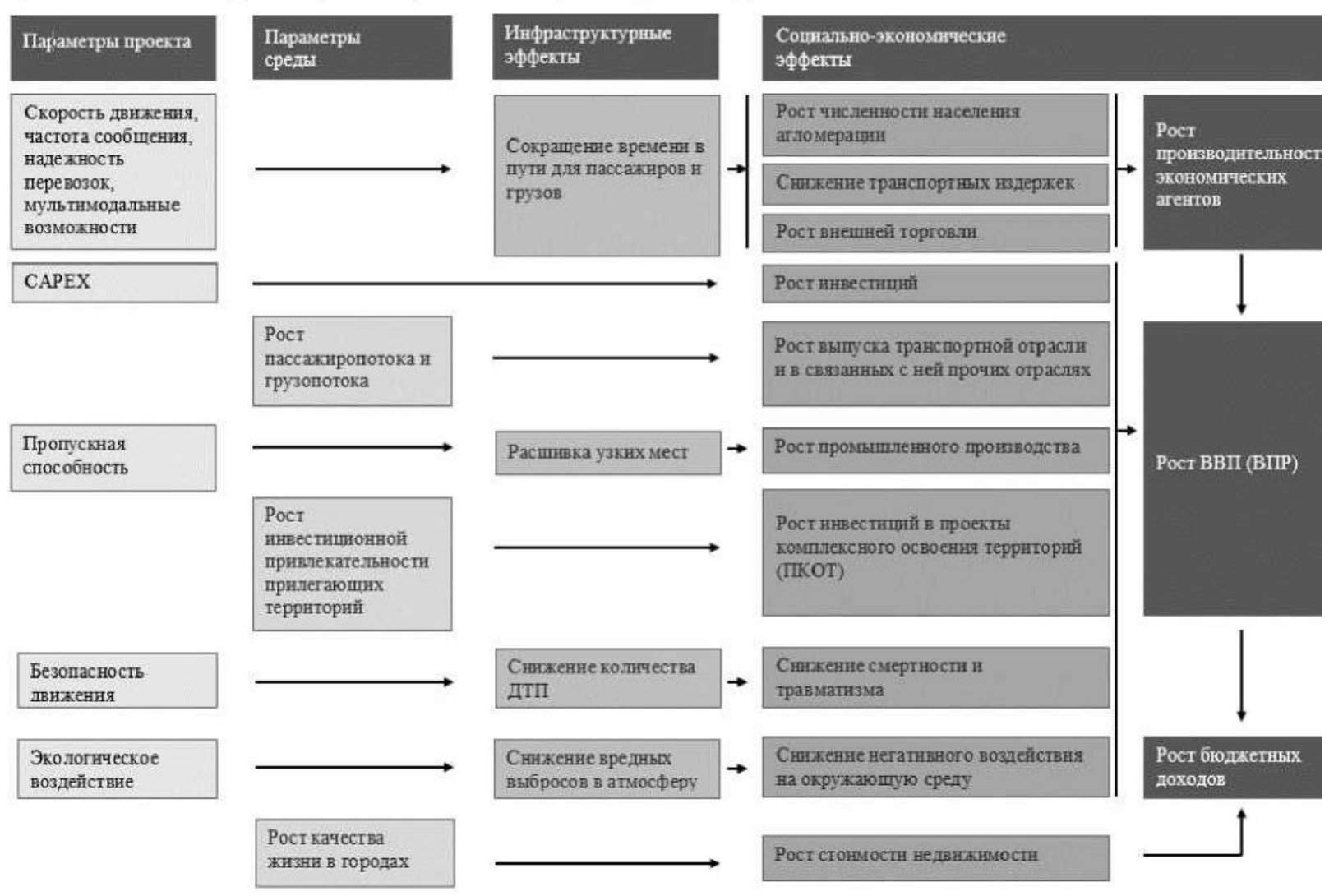

Рисунок 2. Общая схема возникновения социально-экономических эффектов Источник: составлено автором по данным [2]. 
Эффекты инвестиционного спроса не специфичны для транспортных проектов. Они могут возникнуть как на стадии строительства, так и на стадии эксплуатации.

Доводы в пользу крупных транспортных инвестиций часто приводятся с точки зрения влияния на экономические показатели. Существует проблема возникновения несоответствий между результатами проведенных расчетов оценки эффективности социально-экономических эффектов и реальными показателями влияния сферы общественного транспорта на экономические показатели. Расширение количества используемых методик для проведения анализа создает риск использования неблагонадежных аргументов, и соответственно преувеличения результатов проведенного анализа эффектов. Существует необходимость в системе оценки, которая обеспечит учет всех соответствующих воздействий, выявит альтернативные издержки привлечения дополнительных ресурсов к деятельности и удовлетворит потребности различных аудиторий, участвующих в оценке. Необходимо проводить оценку с учетом конкретных условий. Центральным фактором воздействия на экономические показатели является то, как инвестиции частного сектора реагируют на изменения в доступности. Инвестиции в одном месте могут повысить производительность, создать рост, но могут и вытеснить производство и занятость. Таким образом, можно сгруппировать воздействие в рамках концепции по четырем типам: выгоды для пользователей, влияние на производительность влияние на инвестиции и землепользование и влияние на занятость [7].

На сегодняшний день в отечественной практике большим спросом пользуется агломерационный эффект. В случае использования данного эффекта ускорение пассажирского сообщения может быть сопоставлено с бюджетными вложениями. Данный эффект может принести дополнительную пользу за счет расчета, а следовательно, уменьшения времени в пути, что означает увеличение выручки или рост популярности у потребителя.

Пассажиры могут выбирать между автомобилем и общественным транспортом, однако в обоих случаях потребитель может столкнуться с негативным опытом; использование автомобиля приводит к заторам, а общественный транспорт подвержен переполнению. С учетом агломерационных внешних эффектов можно по- лучить следующие результаты: во-первых, агломерационные выгоды подразумевают значительно более низкие тарифы на общественный транспорт и более высокую частоту его движения. Во-вторых, как следствие, агломерационные преимущества оправдывают низкие коэффициенты возмещения затрат в общественном транспорте. В-третьих, что интересно, влияние агломерационных выгод на тарифы и частоту движения общественного транспорта гораздо меньше, если цены на использование дорог экзогенно занижены (цены могут быть занижены в связи с наложением ограничений на ценообразование от регулирующего государственного органа). В этом случае любое смещение заинтересованности потребителя в виде используемого транспорта, вызванное, например, снижением тарифов на общественный транспорт, оказывает противоположный агломерационный эффект на оба вида транспорта (и общий, и личный) [8].

По оценкам различных источников, доля транспорта в общем объеме выбросов составляет порядка 15-25\% [2]. Реализация проектов в области транспорта (изменение инфраструктуры) может привести к изменению в структуре используемых видов транспорта. Например, увеличение количества комфортного общественного транспорта, снижение заторов на специально выделенных для городского транспорта полосах поможет сократить число автомобильных поездок на рассматриваемом маршруте за счет переключения части пассажиров с личного автомобиля на более экологичный вид транспорта.

K ключевым выбросам, обусловленным транспортной деятельностью, относят: воздушные взвеси, окиси азота, углерода, серы, летучие органические соединения. Для оценки влияния выбросов на состояние воздуха используются модели, которые позволяют оценить изменение атмосферы (физическое и химическое) в результате выброса загрязняющих веществ, и модели воздействия (изменение атмосферы в зависимости от типа местности). Также, для оценки экологического ущерба, используются модели, оценивающие последствия загрязнения атмосферы для здоровья людей.

В рамках устойчивого городского планирования был изучен потенциал общественного транспорта для снижения выбросов $\mathrm{CO} 2$, потребления энергии и повышения безопасности дорожного движения. Таким образом, увеличение пассажиров общественного транспорта по 
сравнению с теми, кто передвигается только на автомобиле, стало бы гигантским шагом на пути к более экологически чистым и свободным от стресса городам. В подтверждение данной теории, учеными США был проведен расчет, который показал, что увеличение числа пассажиров общественного транспорта на 9\% в год может привести к сокращению выбросов СО2 на 766000 тонн в год к 2050 году, в то время как увеличение числа пассажиров на 25\% может потенциально сократить кумулятивные выбросы CO2 на 61,3 миллиона тонн [9].

Городской общественный транспорт оказывает непосредственное влияние на социальноэкономическое развитие городских агломераций. При учете плотности и скорости застройки обеспечение каждого жителя личным автомобилем гарантированно приведет к заторам на дорогах, и не сможет в полной мере обеспечить транспортный спрос. Надежное и быстрое сообщение может обеспечить только общественный транспорт. Развитие общественного транспорта в городских агломерациях должно быть ориентировано на следующие запросы потребителей: надежность, доступность, комфорт. При анализе социально-экономических эффектов при реализации проектов развития систем городского общественного транспорта необходимо учитывать следующие показатели: экономия времени пассажиров, сокращение выбросов СО2, сокращение ДТП.

По мнению автора развитие общественного транспорта так же обеспечивает бюджетные эффекты для города. Общественный транспорт обеспечивает развитие прилегающих территорий, что выражается в создании новой недвижимости и рабочих мест. Это обеспечивает рост налоговых поступлений в городской бюджет в виде увеличения сбора налога на имущество физических лиц (из-за роста объема и стоимости м2 недвижимости на прилегающих территориях и сборов налога на доходы физических лиц в силу появления новых рабочих мест как при строительстве объекты городского транспорта, так и при развитии окружающих территорий рядом с объектом).

При модернизации сети общественного транспорта эффект экономии времени в пути может быть достигнут за счет оптимизации логистики, т.е. маршрутной сети общественного транспорта, и повышения скорости сообщения. Данные предложения могут быть реализованы за счет уменьшения интервалов движения транспорта, времени ожидания, создания выделенных полос, и т.п.

Для обеспечения снижения выбросов СО необходима реализация проектов модернизации систем общественного транспорта за счет общего сокращения транспортной работы, выполняемой транспортом с двигателем внутреннего сгорания (ДВС) и увеличения количества электрического транспорта на маршруте, а также сокращения количества транспортных средств с ДВС при повышении доли транспортных средств большого и особо-большого класса.

В процессе перехода отечественной экономики на совершенно новую, инновационную модель управления, становятся особенно актуальными вопросы верных экономических обоснований инвестиций в развитие сферы общественного транспорта. В связи с наличием множества различных методик анализа в данной области, актуальной проблемой становится создание комплексной, мультикритериальной методики оценки эффективности. Для более корректной оценки эффективности развития проектов в отрасли общественного транспорта, необходимо брать во внимание полный спектр социально-экономических эффектов, и их взаимосвязь с экономической, социальной и экологической сферами.

\section{Библиографический список}

1. Петров В.К., Сосяни В.Г., Городской транспорт. Уч. пособие, М.- Л., 1949.

2. Транспортная инфраструктура и экономический рост.-М.Издательство Перо, 2019.-142 с.

3. From business models to value networks and business ecosystems What does it mean for the economics and governance of the transport system? / Pekka Leviakangas [и др.] // Utilities Policy._ 2020._ № 64.— С. 3-12.

4. Public Transport Authorities' use of Cost- Benefit Analysis in practice / Andreas Vigren [и др.] // Research in Transportation Economics. - 2018. - № 69.- C. 560-567.

5. David A.Hensher, Clarifying the complementary contributions of cost benefit analysis and economic impact analysis in public transport investment / David A. Hensher // Bus Transport, Demand, Economics, Contracting, and Policy.-2020. - № 1.- C. 349-363. 
6. State-of-the-art review on multi- criteria decision- making in the transport sector / George Yannis [и др.] // Journal of Traffic and Transportation Engineering (English Edition). - 2020.- T. 7, № 4.- C. 413-431.

7. James J. Laird, Transport investment and economic performance: A framework for project appraisal / James J. Laird, Anthony J. Venables // Transport Policy. - 2017. - № 56.- C. 1-11.

8. Public transport provision under agglomeration economies / Daniel Horcher [и др.] // Regional Science and Urban Economics. - 2020.- № 81.- C. 3-15.

9. Investigating carbon footprint reduction potential of public transportation in United States: A system dynamics approach / Tolga Ercan [и др.] // Journal of Cleaner Production. - 2016. - № 133. - С. 1260-1276. 